Cite this: Phys. Chem. Chem. Phys., 2011, 13, 5556-5560

\title{
Singlet nuclear magnetic resonance of nearly-equivalent spins
}

\author{
Michael C. D. Tayler and Malcolm H. Levitt* \\ Received 27th October 2010, Accepted 23rd January 2011 \\ DOI: $10.1039 / \mathbf{c 0 c p 0 2 2 9 3 d}$
}

\begin{abstract}
Nuclear singlet states may display lifetimes that are an order of magnitude greater than conventional relaxation times. Existing methods for accessing these long-lived states require a resolved chemical shift difference between the nuclei involved. Here, we demonstrate a new method for accessing singlet states that works even when the nuclei are almost magnetically equivalent, such that the chemical shift difference is unresolved. The method involves trains of $180^{\circ}$ pulses that are synchronized with the spin-spin coupling between the nuclei. We demonstrate experiments on the terminal glycine resonances of the tripeptide alanylglycylglycine (AGG) in aqueous solution, showing that the nuclear singlet order of this system is long-lived even when no resonant locking field is applied. Variation of the pulse sequence parameters allows the estimation of small chemical shift differences that are normally obscured by larger $J$-couplings.
\end{abstract}

\section{Introduction}

Nuclear singlet states are non-magnetic states of nuclear spin pairs that may exhibit extraordinarily long lifetimes. ${ }^{1-3}$ In the case of ${ }^{15} \mathrm{~N}$-labeled nitrous oxide, for example, the singlet state of the ${ }^{15} \mathrm{~N}$ pair has a decay time constant of almost 25 minutes in solution. ${ }^{4}$ The long lifetime property suggests applications to the study of slow motions, slow chemical exchange, ${ }^{5-8}$ and the transport of hyperpolarized spin order., 9,10 The rate of singlet decay has also been shown to carry information on the locations of neighboring magnetic nuclei. ${ }^{11}$

Exploiting nuclear singlet order typically requires three different experimental stages, within each of which the resonance frequency difference induced by different chemical shifts plays a key role: (i) nuclear magnetization is converted into nuclear singlet order under the chemical shift asymmetry of the nuclei; (ii) singlet order is then locked by suppressing the chemical shift difference, so preserving it; (iii) the singlet order is converted back to observable nuclear magnetization, again by exploiting the chemical shift difference. The long singlet lifetime is manifested during stage (ii). This requires that singlet-to-triplet interconversion is suppressed, by either transporting the sample into a region of low magnetic field, ${ }^{1,12}$ keeping the sample in high magnetic field while applying resonant radiofrequency irradiation, ${ }^{2,13}$ or switching the symmetry of the molecule using a chemical reaction. ${ }^{10}$

School of Chemistry, University of Southampton, SO17 1BJ, UK.

E-mail: mhl@soton.ac.uk; Fax: + 44 (0)2380593781;

Tel: + 44 (0)2380596753
In this article we explore the situation in which the difference of nuclear resonance frequencies is very small, i.e. near magnetic equivalence. If the chemical shifts of two nuclear sites are so similar that the spin-spin coupling exceeds the difference in chemical shift frequencies, the spin system is said to be 'extremely-strongly-coupled', or 'nearly-equivalent'. The NMR spectrum of nearly-equivalent spin pairs displays a single peak since the spin-spin coupling suppresses the effect of the small chemical shift difference. This situation is found at high magnetic fields when the chemical shifts of two protons are extremely close, and is a rather widespread situation for NMR in low magnetic fields.

Near magnetic equivalence creates both difficulties and opportunities for singlet NMR. Stages (i) and (iii) of the experimental procedure become markedly more difficult in the case of nearequivalence. Until recently, all NMR pulse sequences for converting magnetization into singlet order, and back again, have required a chemical shift frequency difference that is larger than the spin-spin coupling. ${ }^{8}$ Stage (ii), on the other hand, is markedly easier in the case of near-equivalence. It is much easier to suppress a small chemical shift difference than a large one. Indeed, as shown below, no intervention at all is required to sustain the long-lived singlet state in the case of near-equivalence. This is an attractive prospect for certain applications of singlet NMR.

We discuss a technique for stages (i) and (iii) that we have previously used in the context of low-field NMR, where chemical shift frequencies are vanishingly small. ${ }^{14}$ In that case, radiofrequency (RF) pulses applied entirely outside the NMR magnet were used to transform, reversibly, the Zeeman magnetization of a spin pair into nuclear singlet order. The protocol uses 'trains' of spin echoes, with the pulse delay times synchronized with the scalar spin-spin coupling, $J$. Only the difference in the chemical shifts is important for triplet-singlet conversion, since this is the mechanism that connects the symmetric and antisymmetric spin states. Here we show that this method is applicable, generally, to pairs of coupled spins in high field.

\section{Theory}

\subsection{Singlet and triplet mixing}

The singlet and triplet spin quantum states for a pair of spin-1/2 nuclei are defined as

$$
\begin{aligned}
\left|S_{0}\right\rangle=(|\alpha \beta\rangle-|\beta \alpha\rangle) / \sqrt{2} & \left|T_{+1}\right\rangle=|\alpha \alpha\rangle \\
\left|T_{0}\right\rangle=(|\alpha \beta\rangle+|\beta \alpha\rangle) / \sqrt{2} & \left|T_{-1}\right\rangle=|\beta \beta\rangle
\end{aligned}
$$


where the subscripts indicate the values of the projection quantum number, $m$, defined by $\left(I_{1 z}+I_{2 z}\right)\left|\phi_{m}\right\rangle=m \hbar\left|\phi_{m}\right\rangle$. The quantum mechanical evolution in this representation is straightforward to follow because states with different $m$ are isolated from one another, or non-interconverting, since during free evolution the two-spin Hamiltonian

$$
H=\omega_{1}^{0} I_{1 z}+\omega_{2}^{0} I_{2 z}+2 \pi J I_{1} \cdot I_{2}
$$

commutes with the total $z$ angular momentum operator. Here the rotating-frame chemical shift frequencies are defined $\omega_{j}^{0}=\omega^{0}\left(\delta_{j}-\delta_{\text {ref }}\right)$ where $\delta_{j}(j=1,2)$ are the chemical shifts of the two sites and $\delta_{\text {ref }}$ is the chemical shift corresponding to the RF irradiation frequency. $\omega^{0}$ is the nuclear Larmor frequency in the strong magnetic field.

Both the 'outer' triplet states with $|m|=1$ are stationary under free evolution, save for acquiring the time-dependent phases $\exp \left[-\mathrm{i}\left(\pi J-m \omega_{1}^{0}-m \omega_{2}^{0}\right) t\right]$, of their characteristic energies. The states with $m=0$ are the only ones that mix.

In the following we therefore regard $\left|S_{0}\right\rangle$ and $\left|T_{0}\right\rangle$ as a closed two-level quantum system. A state vector, $\left\{\left\langle T_{0} \mid \psi_{0}\right\rangle,\left\langle S_{0} \mid \psi_{0}\right\rangle\right\}$, is defined to represent the superposition- or 'qubit' state, ${ }^{15}$ $\left|\psi_{0}\right\rangle$, for this system.

The evolution of a qubit state may be viewed geometrically in the three-dimensional representation of its Hilbert space known as the Bloch sphere. ${ }^{16}$ The Bloch sphere is a map of all qubits $\{\cos (\xi / 2), \exp (-\mathrm{i} \phi) \sin (\xi / 2)\}$ into points $(\xi, \phi)$ on the surface of a unit sphere (Fig. 1a) so that unitary transformations between qubits are equivalent to rotations (to within a phase factor). A rotation, $R_{n}(\varphi)$, in the Bloch sphere that rotates through an angle $\varphi$ about the axis $\boldsymbol{n}=\left(n_{x}, n_{y}, n_{z}\right)$ can be shown to correspond with the matrix representation

$$
R_{\boldsymbol{n}}(\varphi)=\exp [-\mathrm{i} \varphi \boldsymbol{n} \cdot(X, Y, Z) / 2]
$$

where $E, X, Y$ and $Z$ are the Pauli matrices. ${ }^{16,17}$

$$
\begin{aligned}
& E=\left(\begin{array}{ll}
1 & 0 \\
0 & 1
\end{array}\right) \quad X=\left(\begin{array}{ll}
0 & 1 \\
1 & 0
\end{array}\right) \\
& Y=\left(\begin{array}{cc}
0 & i \\
-i & 0
\end{array}\right) \quad Z=\left(\begin{array}{cc}
1 & 0 \\
0 & -1
\end{array}\right)
\end{aligned}
$$

The $m=0$ qubit evolves according to the $2 \times 2$ matrix representation of eqn (2), which may be expressed as

$$
H \equiv-\frac{\pi J t}{2} E+\frac{\Omega}{2}[(X, Y, Z) \cdot(\sin \theta, 0, \cos \theta)],
$$

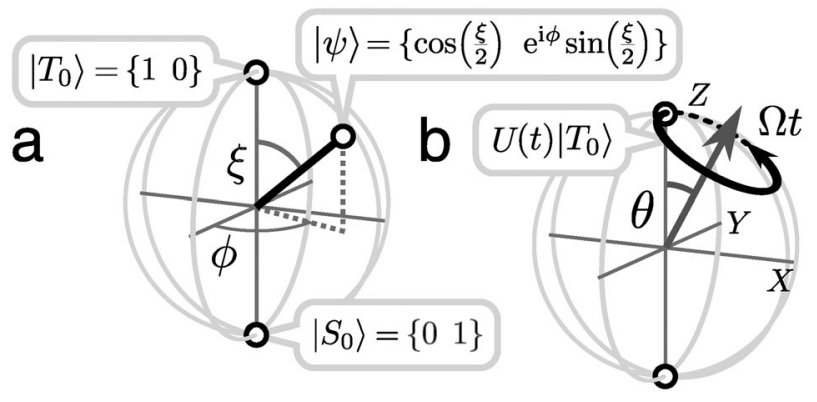

Fig. 1 Bloch vector representation of the $m=0$ qubit, showing time evolution under eqn (2) for the starting state $\left|\psi_{0}\right\rangle=\left|T_{0}\right\rangle$. on introducing the following terms:

$$
\begin{aligned}
& \Omega=\sqrt{(2 \pi J)^{2}+\left(\omega^{0} \Delta \delta\right)^{2}} \\
& \theta=\arctan \left(\omega^{0} \Delta \delta / 2 \pi J\right)
\end{aligned}
$$

The resulting propagator, $U$, on the basis of $\left|S_{0}\right\rangle$ and $\left|T_{0}\right\rangle$ is

$$
\begin{aligned}
U(t) & =\exp [-\mathrm{i} H t] \\
& =\exp [+\mathrm{i} \pi J t / 2] R_{(\sin \theta, 0, \cos \theta)}(\Omega t)
\end{aligned}
$$

for the free evolution. This identifies with a rotation through angle $\Omega t$ about an axis in the ' $X Z$ ' plane, in the Bloch sphere, at azimuth $\theta$ to the vertical (Fig. 1b). Using $\left|\psi_{0}\right\rangle(0)=\left|T_{0}\right\rangle$ this visualization allows one to verify easily that:

- If the nuclei are weakly-coupled (i.e. when $|2 \pi J| \ll\left|\omega^{0} \Delta \delta\right|$ ) then $|\theta| \approx \pi / 2$ and $\Omega \approx \omega^{0} \Delta \delta$; singlet and triplet states interchange at a rate approximately equal to the difference of the nuclear Larmor frequencies.

- For very strongly coupled nuclei (where $\omega^{0} \Delta \delta \ll 2 \pi J$ ), the angle $\theta$ is small and singlet-triplet mixing is suppressed. In this regime it is difficult to achieve transfer between the two states.

- For perfectly magnetically equivalent nuclei, such as in zero magnetic field, or where chemical shifts are coincidental, the angle $\theta$ is zero. The states $\left|S_{0}\right\rangle$ and $\left|T_{0}\right\rangle$ are exact eigenstates and do not interconvert.

\subsection{Resonant spin echo transfer}

Maximum transfer between the singlet and triplet states occurs on leaving a time delay $\pi / \Omega$, but the maximum transfer amplitude is equal to $\sin \theta$ and goes to zero as $\theta \rightarrow 0$. This is unfavourable in the extreme strong coupling situation, for regardless of evolution time the singlet and triplet states do not interconvert to a significant extent.

Singlet-to-triplet mixing may be arranged to accumulate, however, over the course of a spin echo of duration $2 \tau$ (two evolution periods of equal length $\tau$, bisected by a $180^{\circ}$ RF pulse). The qubit state at the end of an echo is determined by the transformation $\boldsymbol{U}(\tau) R_{Z}(\pi) \boldsymbol{U}(\tau)$, where the $180^{\circ} \mathrm{RF}$ pulse leads to a $180^{\circ}$ rotation about the $Z$-axis in the Bloch sphere. This may be seen noting that a $180^{\circ}$ pulse inverts the sign of $\left|T_{0}\right\rangle$, while leaving $\left|S_{0}\right\rangle$ unchanged, and provided the pulse is ideal, there are no transitions between $m=0$ and $|m|=1$. The non-commuting $R_{Z}(\pi)$ and $\boldsymbol{U}(\tau)$ lead to cumulative singlettriplet conversion at the end of the echo. The optimal transfer occurs when $\tau$ is set to $\pi / 2 \Omega$. In this case the propagator is

$$
\boldsymbol{U}(\tau) R_{Z}(\pi) \boldsymbol{U}(\tau)=\mathrm{e}^{(\mathrm{i} \pi J \tau)} R_{(\cos \theta, 0,-\sin \theta)}(2 \theta)
$$

which in the strong-coupling case is approximately a rotation of the Bloch vector through angle $2 \theta$ about the $X$-axis, neglecting the phase factor.

As Fig. 2 shows, $n$ successive echoes generate an overall rotation through the angle $2 n \theta$ in the Bloch sphere. For extremely-strongly coupled spins, the rotation axis is close to the $X$-axis, so $\left|T_{0}\right\rangle \Leftrightarrow\left|S_{0}\right\rangle$ conversion is achievable using back-to-back echoes with intervals $\tau=\pi / 2 \Omega \approx 1 / 4 J$, and the echo number $n$ chosen so that $2 n \theta$ is close to $\pi$. In practice, relaxation losses may limit the maximum achievable transfer.

Regardless of the coupling limit, the mixing rate depends solely on the value of $\omega^{0} \Delta \delta$, not $J$. As expected, the 


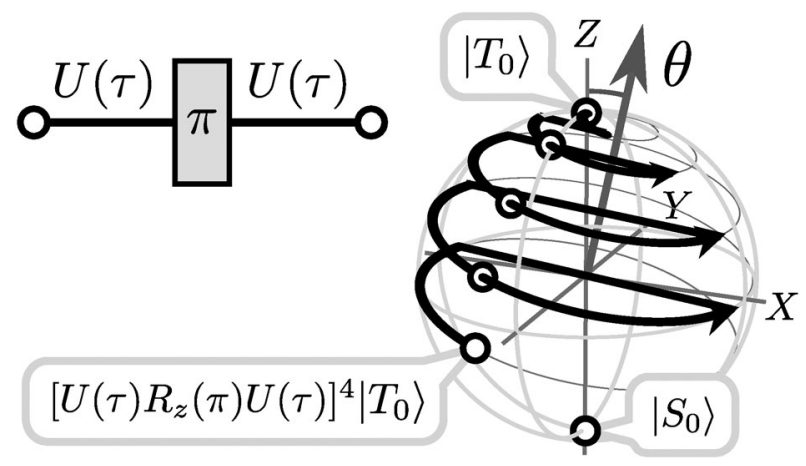

Fig. 2 Singlet and triplet mixing under extreme strong coupling using a synchronized spin echo train. The trajectory of the Bloch vector (thick curve) shows the cumulative conversion of $\left|T_{0}\right\rangle$ into $\left|S_{0}\right\rangle$ due to four echoes, for the optimal interval $\tau=\pi / 2 \Omega$.

singlet-triplet conversion becomes increasingly slow as the nuclei approach magnetic equivalence.

Similar rotations in the $m=0$ Bloch sphere are induced by modulation of the static magnetic field at the $J$-coupling frequency. ${ }^{18}$

\subsection{Preparation of long-lived states}

Conversion between equilibrium Zeeman magnetization and nuclear singlet order was discussed in ref. 14. We shall briefly re-outline the density operator transformations induced by the M2S (Magnetization-to-Singlet) pulse sequence, ${ }^{14}$ which contains two synchronized echo trains (see Fig. 3). To start, we have $\left|S_{0}\right\rangle$ and $\left|T_{0}\right\rangle$ populations equal, with the equilibrium magnetization $I_{z}$ corresponding to population imbalance across the $m= \pm 1$ triplet levels:

$$
|\alpha \alpha\rangle\langle\alpha \alpha|-| \beta \beta\rangle\left\langle\beta \beta|\equiv| T_{+1}\right\rangle\left\langle T_{+1}|-| T_{-1}\right\rangle\left\langle T_{-1}\right|
$$

The initial $90^{\circ}$ ' $y$ ' pulse converts starting magnetization into single-quantum coherences between the inner $(m=0)$ and outer $(|m|=1)$ triplet states, namely

$$
I_{x} \equiv\left|T_{+1}\right\rangle\left\langle T_{0}|+| T_{0}\right\rangle\left\langle T_{+1}|+| T_{0}\right\rangle\left\langle T_{-1}|+| T_{-1}\right\rangle\left\langle T_{0}\right|(12)
$$

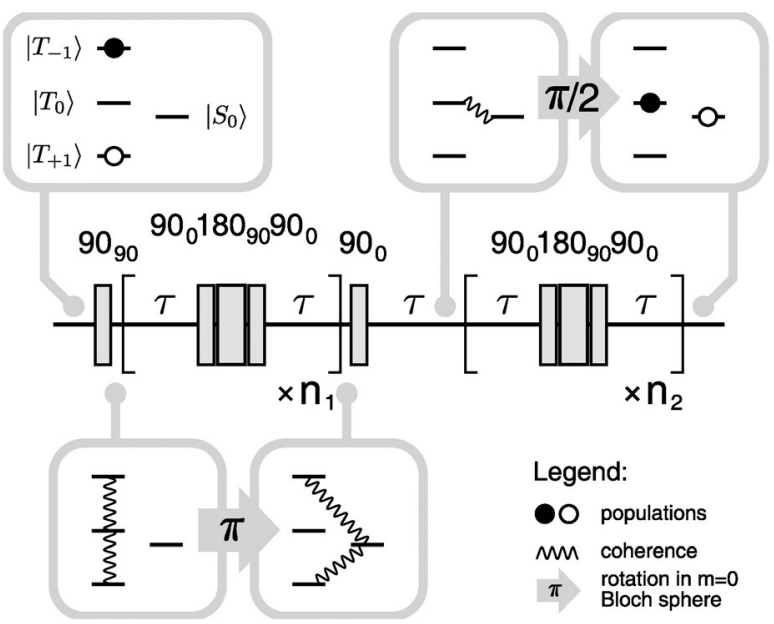

Fig. 3 The 'Magnetization-to-Singlet' (M2S) RF pulse sequence.
These single-quantum coherences are transformed into ones between the singlet and outer triplet states, through a $\pi$ rotation in the Bloch sphere:

$$
i\left(\left|T_{+1}\right\rangle\left\langle S_{0}|+| S_{0}\right\rangle\left\langle T_{+1}|-| S_{0}\right\rangle\left\langle T_{-1}|-| T_{-1}\right\rangle\left\langle S_{0}\right|\right)
$$

which in the Cartesian product operator representation is equal to $\left(I_{1 y}-I_{2 y}\right)$. This conversion exploits a $J$-synchronized spin echo train for approximate interchange of $\left|S_{0}\right\rangle$ and $\left|T_{0}\right\rangle$, so the number of echoes required, $n_{1}$, is the integer such that $2 n_{1} \theta \approx \pi$. As the figure depicts, the $180_{x}^{\circ}$ RF pulses may be implemented as $90_{x}^{\circ} 180_{y}^{\circ} 90_{x}^{\circ}$ composite rotations, to ensure the high accuracy of the inversions required during the pulse train. ${ }^{19}$ The overall phase of the composite pulses is not important in the current context. Single $180^{\circ}$ pulses may be used instead of composite pulses, if the rf field homogeneity is sufficiently good.

A following $90^{\circ}$ ' $x$ ' pulse rotates the transverse magnetization to $\left(I_{1 z}-I_{2 z}\right)$, equivalent to zero-quantum coherence between the $m=0$ states.

$$
i\left(\left|T_{0}\right\rangle\left\langle S_{0}|+| S_{0}\right\rangle\left\langle T_{0}\right|\right) \equiv I_{1 z}-I_{2 z}
$$

Such coherences have been shown, in suitable cases, to be slowly-relaxing compared to $T_{1} \cdot{ }^{20}$ Long-lived coherences (LLCs), as these are known, are conventionally prepared by frequency-selective inversion yet such methods fail when the pair chemical shift difference is unresolved. The above proves a route to LLCs even for very strongly-coupled spin pairs.

The coherences of eqn (14) are finally converted to population difference between the $m=0$ states. Free evolution for time $\tau$ $=1 / 4 J$ first serves to phase shift these coherences, so that they have opposite sign:

$$
\left|T_{0}\right\rangle\left\langle S_{0}|-| S_{0}\right\rangle\left\langle T_{0}\right| .
$$

A $\pi / 2$ rotation in the Bloch space, i.e., the one that transforms $\left|S_{0}\right\rangle \rightarrow\left(\left|S_{0}\right\rangle+\left|T_{0}\right\rangle\right) / \sqrt{2}$, then creates a singlet-triplet population difference:

$$
\left|S_{0}\right\rangle\left\langle S_{0}|-| T_{0}\right\rangle\left\langle T_{0}\right|
$$

In this transformation the appropriate number of echoes, $n_{2}$, should be approximately half $n_{1}$, ensuring $2 n_{2} \theta \approx \pi / 2$.

The evolution of the singlet-triplet population difference is described in ref. 12-14. After equilibration of the triplet populations, the difference between the singlet population and the average of the three triplet populations decays with the time constant $T_{\mathrm{S}}$.

Conversion of singlet order into Zeeman magnetization, the reverse process, should evidently be performed by applying the above transformations in reverse chronological order. The reverse pulse sequence is denoted S2M (Singlet-to-Magnetization). ${ }^{14}$

\section{Results and discussion}

Fig. 4 shows the structure of the tripeptide L-alanyl-glycylglycine (AGG), which contains two isolated $\mathrm{CH}_{2}$ units. The pairs of protons within each of these units are chemically nonequivalent, by virtue of the alanine chiral center that renders the molecule unsymmetrical. The remoteness of the stereocenter, however, generates only a very small chemical asymmetry, as deduced from the one-dimensional spectrum. 


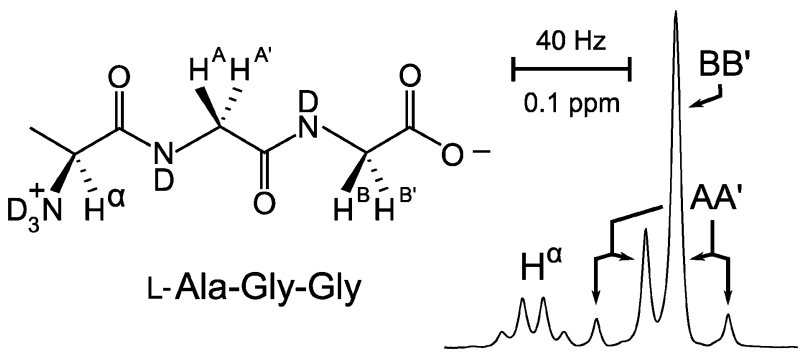

Fig. 4 Proton NMR spectrum of AGG (20 mM in $\left.\mathrm{D}_{2} \mathrm{O}\right), 9.4 \mathrm{~T}$.

Consider the 'central' glycine proton pair, which is only three atom centers away from the chiral center. The two $\mathrm{CH}_{2}$ protons (labeled $\mathrm{AA}^{\prime}$ ) show a characteristic strong-coupling pattern with $\left.\right|^{2} J_{\mathrm{HH}} \mid=18 \mathrm{~Hz}, \omega^{0} \Delta \delta / 2 \pi=14 \mathrm{~Hz}$, corresponding to a chemical shift difference $\Delta \delta=\left(\delta_{1}-\delta_{2}\right)$ of $0.035 \mathrm{ppm}$. The terminal $\mathrm{CH}_{2}$ resonance appears as just a single peak with the chemical shifts of the nuclei unresolved (coincidentally, the resonance lies on top of one of the peaks for the other diastereotopic pair). These protons (labeled $\mathrm{BB}^{\prime}$ ) are 'nearly equivalent' because the chiral center is remote, six sigma bonds away along the peptide chain. The $\mathrm{AA}^{\prime}$ and $\mathrm{BB}^{\prime}$ spin pairs both have a $T_{1}$ of $\approx 0.75 \mathrm{~s}$, as measured by saturation recovery at $9.4 \mathrm{~T}$ on a degassed solution.

We have detected singlet order on the pair $\mathrm{BB}^{\prime}$ by using the pulse sequences shown in Fig. 5a and b. At a field of $9.4 \mathrm{~T}$, Zeeman order was transported into singlet-triplet population difference using the resonant echo method described through eqn (11)-(16). Following excitation, the state was left for a time delay, for relaxation (see below). Remanent singlet-triplet order after this period was reconverted to in-phase magnetization, which is estimated through the spectral intensity. The plots in Fig. 5 show the peak amplitude as a function of the relaxation delay, plus the fitted exponential time constants. A two-step phase cycle $\left\{0^{\circ}, 180^{\circ}\right\}$ on both the starting $90^{\circ}$ pulse and on the receiver was used to eliminate signals arising from longitudinal recovery during the relaxation delay.

The absence of spin locking does not quench the long lifetime of the singlet state of the extremely-strongly coupled pair (Fig. 5a). We measured a singlet decay constant $T_{\mathrm{S}}=$ $11.5 \pm 0.2$ seconds which is approximately 15 times longer than $T_{1}$. This result demonstrates that unlike for weakly-coupled spin pairs it is not necessary to force coherent isolation of the singlet state using RF spin-locking, in the nearly-equivalent case. Coherent evolution between singlet and triplet states is already suppressed by the internuclear $J$ coupling, as predicted by the theory of ref. 13 .

The singlet relaxation constant, $T_{\mathrm{S}}$, was also measured in the presence of RF locking, after leaving 3.0 seconds $\left(\approx 4 T_{1}\right)$ for the equilibration of triplet populations (Fig. 5b). We used a WALTZ-16 modulation of the applied irradiation at a nutation frequency of $2.0 \mathrm{kHz}$, which is sufficiently-strong to maintain long-lived singlet population with the carrier placed up to $\pm 2.0 \mathrm{kHz}$ off resonance. ${ }^{13}$ Fitting yielded $T_{\mathrm{S}}=17.0 \pm$ 0.5 seconds for the $\mathrm{BB}^{\prime}$ pair. Singlet relaxation therefore increases to around 23 times $T_{1}$ when the small chemical shift difference is suppressed by a spin-locking field.

The spin echo train parameters used in these experiments were $\tau=13.9 \mathrm{~ms}$ and $n_{1}=6, n_{2}=3$, which were found

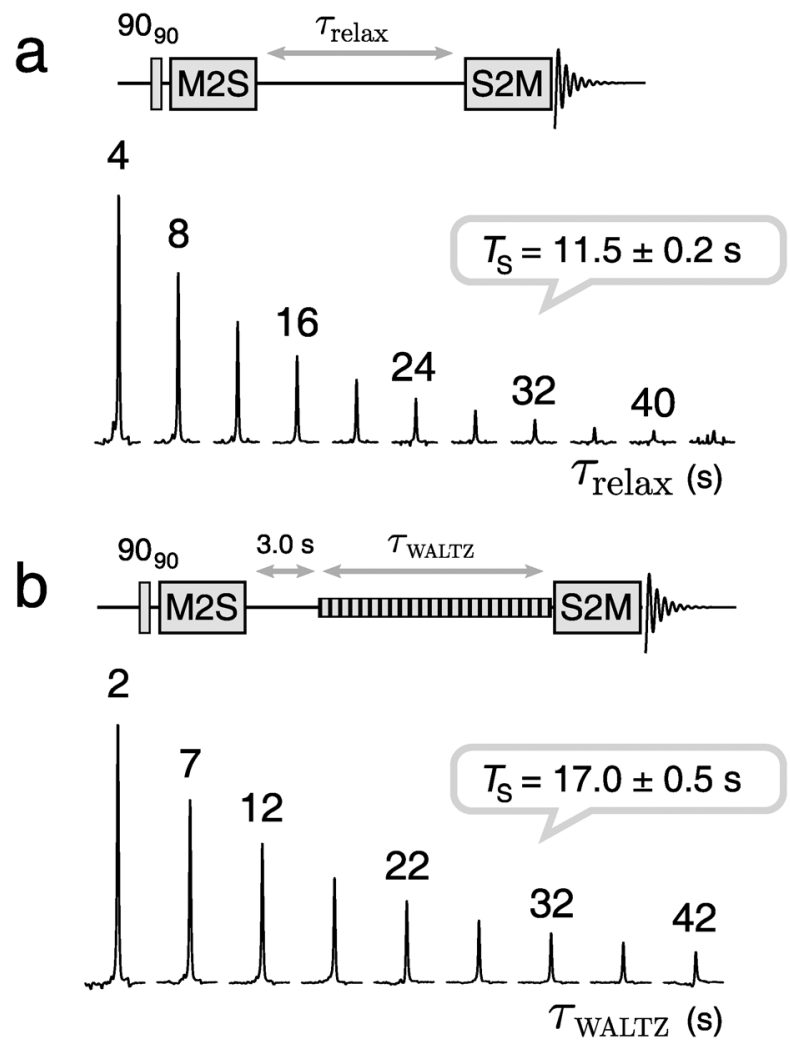

Fig. 5 Singlet relaxation of the extremely-strongly coupled $\mathrm{BB}^{\prime}$ proton pair in AGG: (a) when no RF locking is applied; (b) during forced magnetic equivalence under an RF field. An additional delay of 3 seconds is provided in (b) in order to allow equilibration of the triplet populations. Blocks 'M2S' and 'S2M' in the pulse diagrams abbreviate the forward and reverse 'Magnetization to Singlet' transformations, respectively.

empirically to give maximum Zeeman-to-singlet population transfer. Variation of each of these parameters in turn about this maximum excitation is shown in Fig. 6, noting that the
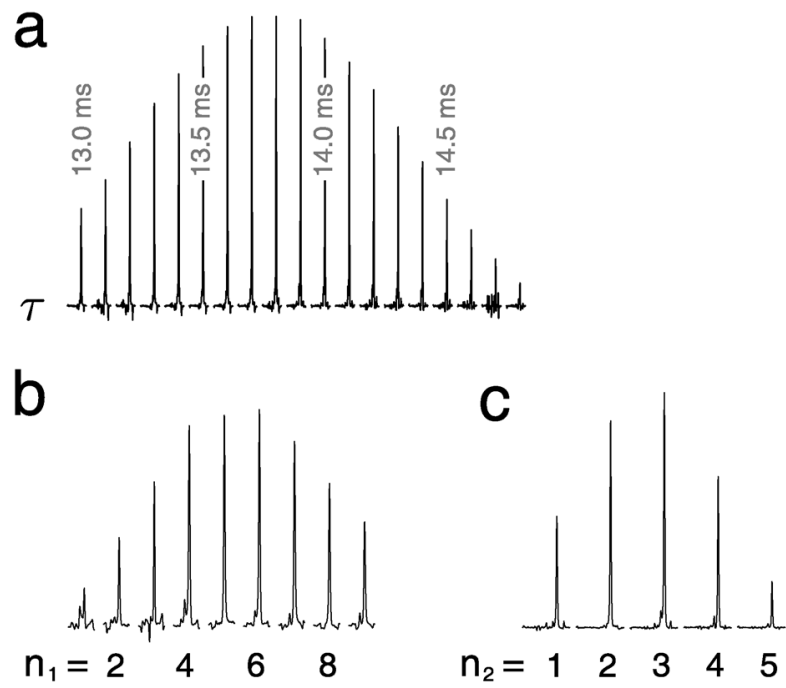

Fig. 6 Efficiency of Magnetization-to-Singlet transfer. Shown are the amplitudes of the NMR signal from the sequence in Fig. 5a following variation of: (a) the spin echo delay, $\tau$; the number of echoes performed (b) $n_{1}$, in the first train and (c) $n_{2}$, in the second. Each displayed spectral region has a width of $100 \mathrm{~Hz}$. 
adjustments are made for both the preparation and reconversion parts of the experimental sequence. With reference to $\Omega$ and $\theta$ in eqn (6) and (7) we have determined values for the chemical shift separation of the pair: $\omega^{0} \Delta \delta / 2 \pi=5.0 \pm 0.1 \mathrm{~Hz}(\Delta \delta \approx 12 \mathrm{ppb})$ and the geminal coupling constant ${ }^{2} J_{\mathrm{BB}^{\prime}}=18.0 \pm 0.05 \mathrm{~Hz}$. This $\mathrm{BB}^{\prime}$ coupling constant is very similar to that of the $\mathrm{AA}^{\prime}$ spin pair.

\section{Conclusions}

Nuclear singlet order may be excited on pairs of protons in spite of a chemical shift difference many times smaller than the spin-spin scalar coupling. When the chemical shifts are so similar, the coherent evolution between singlet and triplet spin states is suppressed to the extent that very long lived singlet order can persist in high field in the absence of RF irradiation. This is an attractive prospect for in vivo magnetic resonance imaging (MRI) studies using hyperpolarized material. ${ }^{9,10}$ The use of nearlyequivalent spin pairs offers an alternative to the use of chemical reactions for making and breaking molecular symmetry. ${ }^{10}$

The precise determination of small and unresolved chemical shift differences, of order parts-per-billion, may also prove useful in other contexts, for instance in measuring isotope shielding effects. ${ }^{21}$

\section{Acknowledgements}

This research was funded by EPSRC (UK). We are grateful to G. Pileio and M. Carravetta, for discussions, and O. G. Johannessen, for experimental help.

\section{References}

1 M. Carravetta, O. G. Johannessen and M. H. Levitt, Phys. Rev. Lett., 2004, 92, 153003.
2 M. Carravetta and M. H. Levitt, J. Am. Chem. Soc., 2004, 126, 6228-6229.

3 M. H. Levitt, Singlet and Other States with Extended Lifetimes, Encyclopedia of Magnetic Resonance, John Wiley \& Sons, Ltd, 2010, vol. 9.

4 G. Pileio, M. Carravetta, E. Hughes and M. H. Levitt, J. Am. Chem. Soc., 2008, 130, 12582-12583.

5 P. Ahuja, R. Sarkar, P. R. Vasos and G. Bodenhausen, J. Am. Chem. Soc., 2009, 131, 7498-7499.

6 S. Cavadini, J. Dittmer, S. Antonijevic and G. Bodenhausen, J. Am. Chem. Soc., 2005, 127, 15744-15748.

7 R. Sarkar, P. Ahuja, P. R. Vasos and G. Bodenhausen, ChemPhysChem, 2008, 9, 2414-2419.

8 R. Sarkar, P. R. Vasos and G. Bodenhausen, J. Am. Chem. Soc., 2007, 129, 328-334.

9 P. R. Vasos, A. Comment, R. Sarkar, P. Ahuja, S. Jannin, J. P. Ansermet, J. A. Konter, P. Hautle, B. van den Brandt and G. Bodenhausen, Proc. Natl. Acad. Sci. U. S. A., 2009, 106 18469-18473.

10 W. S. Warren, E. Jenista, R. T. Branca and X. Chen, Science, 2009, 323, 1711-1714.

11 M. C. D. Tayler, S. Marie, A. Ganesan and M. H. Levitt, J. Am. Chem. Soc., 2010, 132, 8225-8227.

12 M. Carravetta and M. H. Levitt, J. Chem. Phys., 2005, 122, 214505.

13 G. Pileio and M. H. Levitt, J. Chem. Phys., 2009, 130, 214510.

14 G. Pileio, M. Carravetta and M. H. Levitt, Proc. Natl. Acad. Sci. U. S. A., 2010, 107, 17135-17139.

15 D. P. DiVincenzo, Science, 1995, 270, 225-261.

16 R. Shankar, Principles of Quantum Mechanics, Plenum Press, New York, 2nd edn, 1994.

17 D. Varshalovich, A. Moskalev and V. Khersonskii, Quantum Theory of Angular Momentum, World Scientific, Singapore, 1988.

18 G. Pileio, M. Carravetta and M. H. Levitt, Phys. Rev. Lett., 2009, 103, 083002

19 M. H. Levitt, Prog. Nucl. Magn. Reson. Spectrosc., 1986, 18, 61-122.

20 R. Sarkar, P. Ahuja, P. R. Vasos and G. Bodenhausen, Phys. Rev. Lett., 2010, 104, 053001.

21 P. E. Hansen, Prog. Nucl. Magn. Reson. Spectrosc., 1988, 20, 207-255. 